\title{
ESTABILIZACIÓN DE LA BOSTA DE CABALLO MEDIANTE UN PROCESO AERÓBICO SIMILAR AL COMPOSTAJE
}

\author{
Lena A. Tellez Monzón ${ }^{* a, b}$, Luis F. Ortiz Dongo ${ }^{b}$, José L. Calle Maravíc, \\ Paola A. Jorge Montalvo ${ }^{\mathrm{a}, \mathrm{b}}$, Mary F. Cesare Coral ${ }^{\mathrm{a}, \mathrm{b}}$, Lizardo Visitación Figueroa ${ }^{\mathrm{a}, \mathrm{b}}$
}

\section{RESUMEN}

El presente estudio evaluó la estabilización de la bosta de caballo (mezcla de cascarilla de arroz, estiércol y orines de caballo), mediante un proceso aeróbico similar al compostaje. El proceso de estabilización fue evaluado en dos pilas de bosta de caballo durante 16 semanas tomando muestras cada semana. La disminución de la temperatura de 66,7 a $41,0{ }^{\circ} \mathrm{C}$, la reducción de la densidad de 394,0 a 370,4 kg/m3, la reducción de la capacidad de retención de agua de 220,7 a $125,6 \%$ y la reducción de la actividad respiratoria de 9,8 a menos de $2 \mathrm{mgO}_{2} / \mathrm{g} \mathrm{ms} / \mathrm{d}$, fueron indicativos de la estabilización de la bosta de caballo, el material fácilmente degradable de estiércol y orines fueron removidos por degradación aeróbica y lavados con el riego, quedando solo cascarilla de arroz con una fracción mínima de otros materiales. Por otro lado, el incremento del nitrógeno total y descenso de la relación $\mathrm{C} / \mathrm{N}$ al final de la semana 16 fueron indicativos de la fijación del nitrógeno por microorganismos adheridos en la cascarilla de arroz. La estabilización y la fijación de microorganismos en el material residual de la bosta de caballo hacen de este material un buen soporte para la degradación de material orgánico fácilmente biodegradable.

Palabras clave: Bosta de caballo, estabilización, proceso aeróbico, cascarilla de arroz

\section{STABILIZATION OF THE HORSE MANURE THROUGH AN AEROBIC PROCESS SIMILAR TO COMPOSTING}

\begin{abstract}
In the present study, the stabilization of horse manure (mixture of rice husk, manure and horse urine) was evaluated through an aerobic process similar to composting. The stabilization process was evaluated in two piles of horse manure for 16 weeks, taking samples every week. A decrease in temperature from 66,7 to $41,0{ }^{\circ} \mathrm{C}$, the reduction of the density from 394,0 to $370,4 \mathrm{~kg} / \mathrm{m} 3$, the reduction of the water retention capacity from 220,7 to $125,6 \%$ and the reduction of the respiratory activity of 9.8 to less than $2 \mathrm{mgO} 2 / \mathrm{g} \mathrm{ms} / \mathrm{d}$, were indicators of the stabilization of the horse manure, the easily degradable material of manure and urine

\footnotetext{
a Centro de Investigación en Química, Toxicología y Biotecnología Ambiental - Facultad de Ciencias - Universidad Nacional Agraria La Molina, Lima - Perú ltellez@lamolina.edu.pe

b Centro Modelo de Tratamiento de Residuos - Universidad Nacional Agraria La Molina, Lima - Perú

c Lab. de Energía Renovables - Facultad de Ingeniería Agrícola - Universidad Nacional Agraria La Molina, Lima - Perú
} 
were removed by aerobic degradation and washed with irrigation, leaving only the rice husk with a minimum fraction of other materials. On the other hand, the increase in total nitrogen and a decrease in the $\mathrm{C} / \mathrm{N}$ ratio at the end of the week 16 were indicative of the nitrogen fixation by microorganisms adhered to the rice husk. The stabilization and the fixation of microorganisms in the residual material of the horse manure, make this material a good support for the degradation of easily biodegradable organic material.

Key words: Horse manure, stabilization, aerobic process, rice husk.

\section{INTRODUCCIÓN}

La bosta de caballo (BC) es un residuo agropecuario formado por cascarilla de arroz (20\%), orines $(50 \%)$ y estiércol de caballo (30\%), que en promedio pueden generarse $30 \mathrm{~kg} /$ día. caballo $^{1}$. Esta combinación de materiales de fácil biodegradación (estiércol y orines) con materiales de difícil degradación como la cascarilla de arroz por su alto contenido de lignina, entre $19-25 \%$, 2,3 , pueden ser utilizados para la obtención de un sustrato estable enriquecido con microoganismos, que pueda ser reutilizado en la degradación de residuos biodegradables en forma continua.

Los componentes de la $\mathrm{BC}$ presentan relaciones $\mathrm{C} / \mathrm{N}$ entre 20 a $>100$ para cascarilla de arroz, 22 a 50 para estiércol de caballo y $<1$ para la orina de caballo ${ }^{4,5,6}$. En el proceso de estabilización de este material la cascarilla de arroz no se descompone, quedando un material con una relación $\mathrm{C} / \mathrm{N}$ estimado de 12 , este material al tener un valor bajo de $\mathrm{C} / \mathrm{N}$ en relación al óptimo, entre 25 a 30, para el proceso de estabilización similar al compostaje ${ }^{7}$, es susceptible a pérdidas de nitrógeno por volatilización del NH3, en especial cuando se exceden los requerimientos nutricionales de los microoganismos, esto a concentraciones superiores a $2,4 \%$ de nitrógeno ${ }^{8,9}$.

La emisión del amoniaco es causada por la amonización del nitrógeno orgánico (proteínas, aminoácidos entre otros) en la fase termofílica ${ }^{10}$. Los nitratos ausentes en la fase termofílica debido a las altas temperaturas y excesivas concentraciones de amonio que inhiben las bacterias nitrificantes, se forman en la fase mesofílica debido a la oxidación del nitrógeno amoniacal en exceso ${ }^{7,11}$. En la estabilización de BC la gran concentración de nitratos es removido por procesos de riego debido a que la cascarilla de arroz no tiene una buena retención de agua. En la fase de maduración del material estabilizado se produce la fijación del nitrógeno en microorganismos de la materia orgánica resultante ${ }^{12}$, ocasionando una disminución en la relación $\mathrm{C} / \mathrm{N}$ por incremento del nitrógeno. Por otro lado la relación $\mathrm{C} / \mathrm{N}$ en el proceso de estabilización disminuye como resultado de la degradación de la materia orgánica y liberación de dióxido de carbono principalmente en la fase termofilica ${ }^{13}$.

La densidad aparente de la BC se encuentra entre $50 \mathrm{~kg} / \mathrm{m}^{3}$ y $750 \mathrm{~kg} / \mathrm{m}^{3}$, corresponde a material suelto donde la comprensibilidad del material es el factor determinante como lo sugieren estudios anteriores ${ }^{14,15}$. Durante el proceso de estabilización el material degradado 
se va asentando disminuyendo los espacios aéreos ${ }^{15,16}$. La densidad puede disminuir por lavados sucesivos de la materia orgánica y poca capacidad para retener agua del material resultante, esto genera un material con elevado contenido de espacios aéreos que puede ser utilizado posteriormente en la degradación de material orgánico.

La capacidad de retención de agua (CRA) es parámetro importante para el proceso de estabilización debido a que permite la retención temporal del agua y su posterior liberación por lixiviación o evaporación. Para la mezcla de estiércol y cascarilla de arroz puede alcanzar valores de entre 105 a $280 \%{ }^{17,18}$. La CRA en el material estabilizado puede descender debido a la perdida de la materia orgánica biodegradada por lavados y cuando solo permanece la cascarilla de arroz ${ }^{17}$.

La actividad respiratoria es un parámetro importante para determinar la estabilidad de la BC, estudios realizados para cáscara de arroz y mezcla de cáscara de arroz con estiércol vacuno presentaron valores de 5 y $21 \mathrm{mgO} 2 / \mathrm{g} \mathrm{ms} / \mathrm{d}$, respectivamente, con una humedad de $40 \%{ }^{17}$. La cascarilla de arroz después de la pérdida de materia orgánica por degradación y lavado de pila, genera un material muy estable a procesos degradativos aeróbicos según el índice de madurez de la CCQC $<3 \mathrm{mgO} 2 / \mathrm{g} \mathrm{ms} \mathrm{d}^{19,20}$.

El presente trabajo tuvo como objetivo evaluar el proceso de estabilización de $\mathrm{BC}$ durante un proceso aeróbico similar al compostaje, logrando obtener un producto estable y enriquecido de microorganismos que puedan degradar otros materiales orgánicos.

\section{PARTE EXPERIMENTAL}

\section{Proceso de estabilización}

Los residuos de bosta de caballo (BC) conformados por excretas de caballo, orines y cascarilla de arroz fueron recolectados de un hipódromo en Lima, estos fueron estabilizados en las instalaciones del CEMTRAR de la Universidad Nacional Agraria La Molina, la estabilización fue realizada en dos pilas triangulares de $10 \mathrm{~m}$ de largo, $3 \mathrm{~m}$ de ancho y 1,5 $\mathrm{m}$ de altura denominadas E-1 y E-2. Se promovió la actividad degradativa de las excretas y orines por los microorganismos autóctonos de la mezcla, para lo cual se mantuvo la humedad de las pilas entre 40 a $60 \%$, se realizó volteos semanales en forma manual asegurando la buena ventilación de las pilas durante 16 semanas.

\section{Muestreo y métodos analíticos}

Se obtuvieron muestras homogéneas y representativas del material con el método "Coning and Quartering Procedure"21, con una frecuencia semanal. Los métodos analíticos empleados para la determinación de la temperatura, densidad, capacidad de retención de agua, carbono orgánico y nitrógeno total, fueron los sugeridos por la ordenanza de compost de la BGBI. II 292, 200122. La determinación de la actividad respiratoria fue realizada con el método sugerido por la ONORM 2027-4:2012 y adaptado al método sugerido por otros autores ${ }^{17}$. 


\section{RESULTADOS Y DISCUSIÓN}

\section{Temperatura}

En el proceso de estabilización de la $\mathrm{BC}$ la fase termofílica se realiza en los tres primeros días, alcanzando temperaturas de $66,7^{\circ} \mathrm{C}$, lo que indica un rápido inicio de la actividad microbiana y se mantuvo hasta el día 14 similar a lo reportado por otros estudios ${ }^{7,13}$. Los materiales de fácil biodegración como el estiércol y orines son degradados rápidamente. La fase mesofílica se inicia después del día 15 hasta el día 42. En la fase de maduración la temperatura se mantuvo alrededor a $41^{\circ} \mathrm{C}$, esto es un indicativo que el proceso degradativo se ha terminado por disminución del material orgánico biodegradable ${ }^{20,23}$, como se observa en la figura 1.

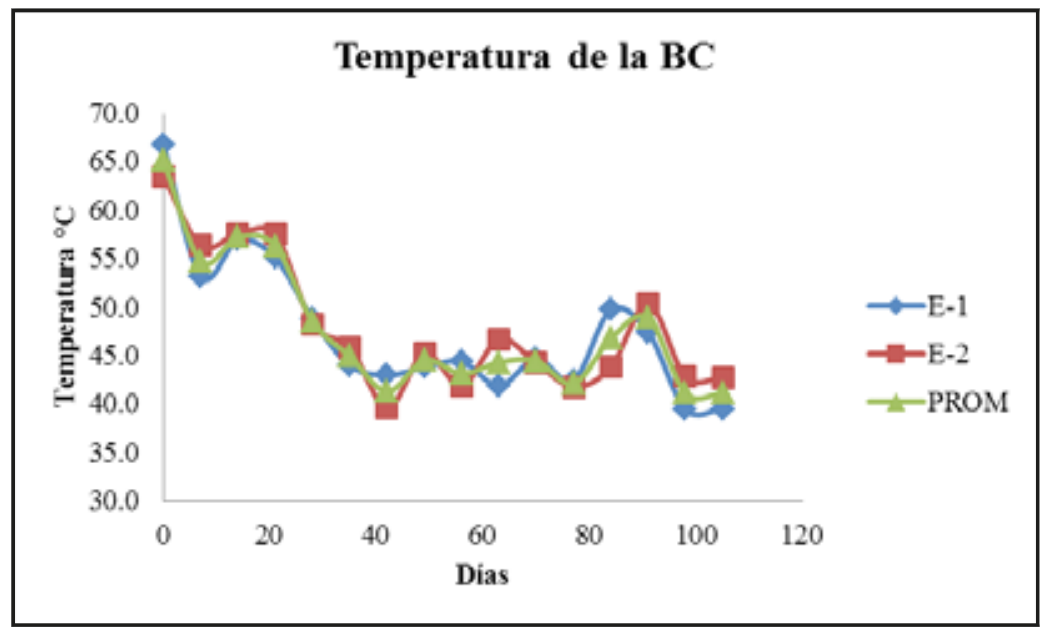

Figura 1. Cambios en la temperatura durante el proceso de estabilización

\section{Densidad aparente}

La densidad aparente inicial de la BC fue $394 \mathrm{~kg} / \mathrm{m}^{3}$, este valor se encuentra dentro del rango esperado para una mezcla de cascarilla de arroz $50 \mathrm{~kg} / \mathrm{m}^{3}$ y estiércol $750 \mathrm{~kg} / \mathrm{m}^{3,14}$, el valor inicial corresponde a material suelto donde la comprensibilidad del material es el factor determinante ${ }^{15}$. Durante el proceso degradativo aeróbico se alcanzan valores máximos de $689,1 \mathrm{~kg} / \mathrm{m}^{3}$ en las seis primeras semanas, esto debido a que el material degradado se va asentando disminuyendo los espacios aéreos, similar a lo obtenido durante 28 días para compost de paja y algas $\left(310\right.$ a $\left.670 \mathrm{~kg} / \mathrm{m}^{3}\right)$ y para densidad aparente seca de paja y estiércol de pollo ${ }^{15,16}$. Después de la semana ocho el valor de la densidad se reduce hasta alcanzar valores mínimos de $370,4 \mathrm{~kg} / \mathrm{m}^{3}$, este cambio se debe a la pérdida de la materia orgánica por lavados y poca capacidad para retener agua del material resultante consistente de cascarilla de arroz, como se observa en la figura 2. 


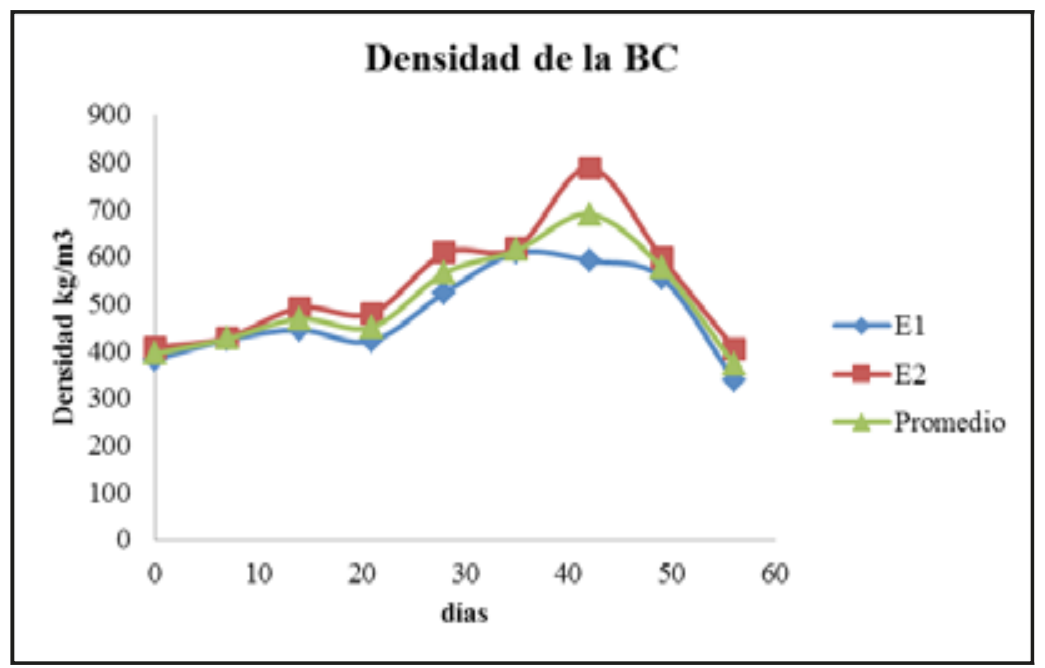

Figura 2. Cambios en la densidad durante el proceso de estabilización

\section{Capacidad de retención de agua (CRA)}

La capacidad de retención de agua dentro de las dos primeras semanas del tratamiento aeróbico de la BC alcanza valores de hasta $220,7 \%$, estos valores son similares a los reportados para la mezcla de estiércol vacuno y cascarilla de arroz $280,0 \%$ y superiores a los reportados para biosólidos así como para diversos tipos de estiércoles $105-149 \%{ }^{17,18}$. La CRA desciende a partir de la tercera semana hasta valores de $125,6 \%$ al cabo de 12 semanas, esto nos sugiere que en la mezcla solo queda la cascarilla de arroz cuyo valor reportado por otro estudio fue de $170,0 \%{ }^{17}$. Al igual que en el caso de la densidad la pérdida de materia orgánica por degradación y lavado de la pila, se manifiesta en una reducción de la CRA, como se observa en la figura 3.

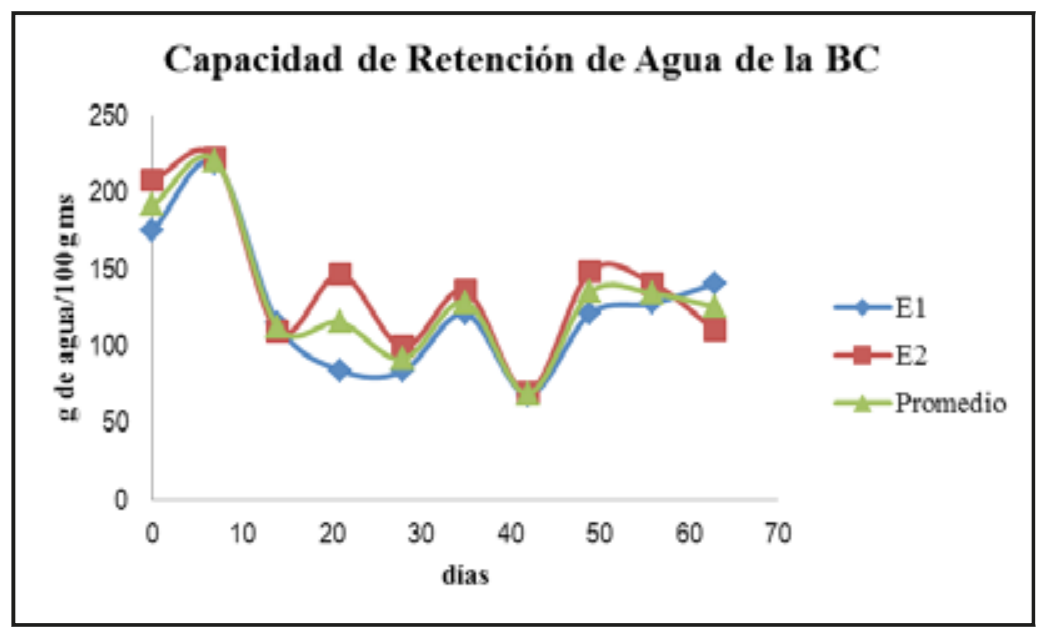

Figura 3. Cambios en la CRA durante el proceso de estabilización 


\section{Actividad respiratoria}

La actividad respiratoria de la bosta de caballo en las dos primeras semanas alcanza valores máximos de hasta $9,8 \mathrm{mgO}_{2} / \mathrm{g} \mathrm{ms} / \mathrm{d}$, este valor es menor al reportado para cascarilla de arroz y estiércol vacuno de $21,0 \mathrm{mgO}_{2} / \mathrm{g} \mathrm{ms} / \mathrm{d}$ con una humedad de $40 \%{ }^{17}$, debido a la presencia de material orgánico degradable, principalmente constituido por el estiércol de caballo. Al finalizar las 16 semanas la actividad respiratoria disminuye hasta valores mínimos menores a 2,0 $\mathrm{mgO}_{2} / \mathrm{g} \mathrm{ms} / \mathrm{d}$, correspondiente a la cascarilla de arroz remanente, similar a lo reportado para la cascarilla de arroz 5,0 $\mathrm{mgO}_{2} / \mathrm{g} \mathrm{ms} / \mathrm{d}$ con una humedad de $40 \%{ }^{17}$. La pérdida de materia orgánica por degradación y lavado de la pila generan un material de cascarilla de arroz muy estable a procesos degradativos aeróbicos, según el índice de madurez de la CCQC $<3,0 \mathrm{mgO} 2 / \mathrm{g} \mathrm{ms} / \mathrm{d}^{19,20}$, como se observa en la figura 4 .

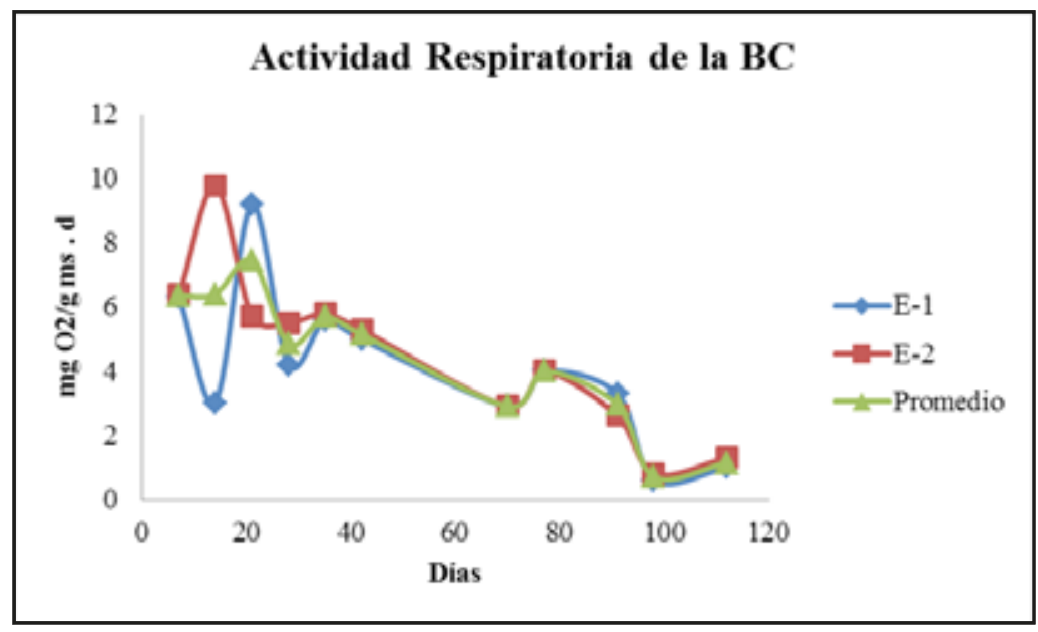

Figura 4. Cambios en la actividad respiratoria durante el proceso de estabilización

\section{Nitrógeno total}

El contenido inicial promedio del nitrógeno en la BC fue de 1,42\%, este corresponde a fuentes nitrogenadas principalmente del estiércol y orines del caballo así como de la cascarilla de arroz. Se alcanza valores máximos de 1,99\% entre los 30 y 40 días debido a la oxidación de la materia orgánica y liberación del dióxido de carbono que reduce el porcentaje de carbono en el material ${ }^{7}$. Después del día 60 se observa una disminución del contenido de nitrógeno hasta valores por debajo de 1,21\%, esto sugiere pérdidas de las formas nitrogenadas como los nitratos y amonio, por procesos de lavado y evaporación, quedando básicamente en la bosta solo cascarilla de arroz de difícil degradación bajo las condiciones de estabilización. Después del día 90 se observa un ligero incremento de nitrógeno hasta valores de 1,30\% probablemente debido a la fijación del nitrógeno en microorganismos de la materia orgánica resultante ${ }^{12}$, como se observa en la figura 5. 


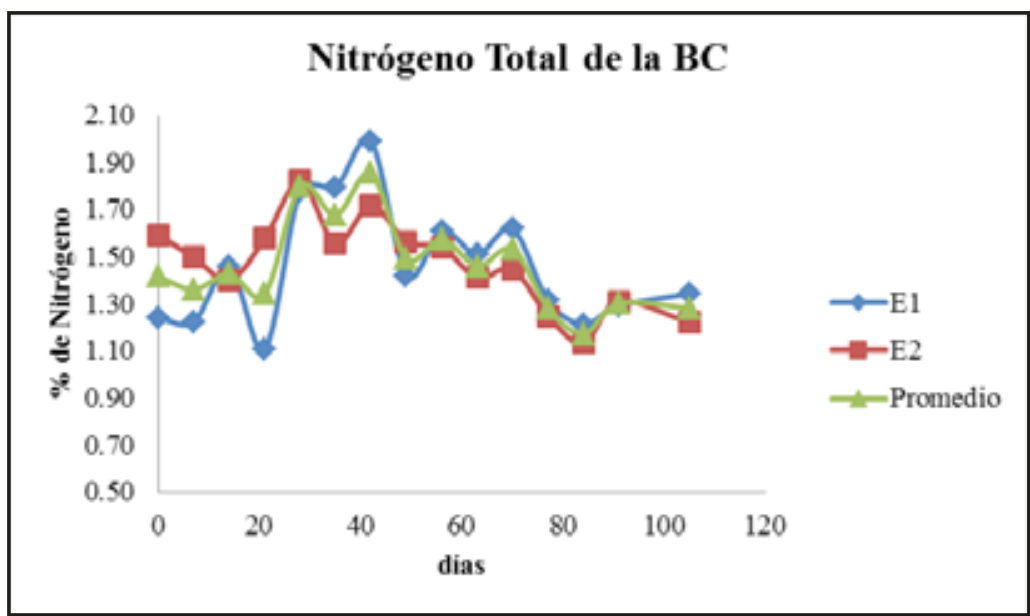

Figura 5. Cambios del porcentaje de nitrógeno durante el proceso de compostaje

\section{Relación C/N}

En el proceso de estabilización de $\mathrm{BC}$ la relación $\mathrm{C} / \mathrm{N}$ disminuye dentro de los 20 primeros días de 17,8 hasta 13,8, como resultado de la degradación de la materia orgánica provenientes del estiércol y orines de caballo y liberación de dióxido de carbono en la fase termofílica ${ }^{13}$. Después del día 60 la relación $\mathrm{C} / \mathrm{N}$ en el material estabilizado de $\mathrm{BC}$ se incrementa hasta 21,5 , esto es debido a que el nitrógeno mineralizado en forma de nitratos o iones amonio por efectos del lavado, es removido, quedando en el material principalmente residuos de cascarilla de arroz. A partir del día 90 se observa una disminución de la relación hasta 18,7 por incremento del nitrógeno que se fija en los microorganismos ${ }^{12}$, como se observa en la figura 6.

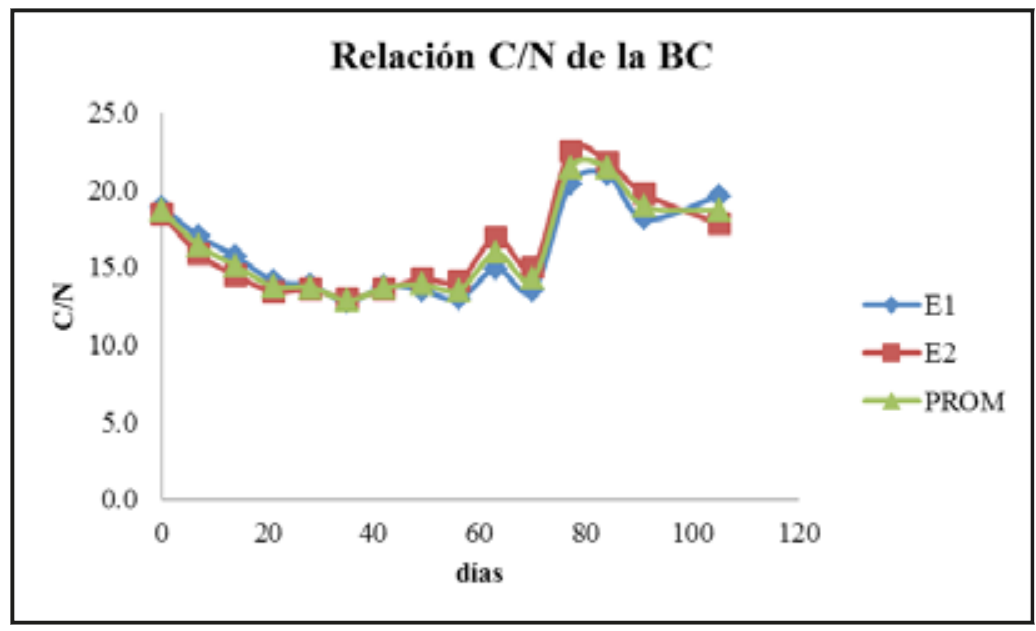

Figura 6. Cambios de la relación $\mathrm{C} / \mathrm{N}$ durante el proceso de compostaje 


\section{CONCLUSIONES}

La bosta de caballo en un periodo de cuatro meses puede ser estabilizado utilizando un tratamiento aeróbico similar al compostaje, generando como resultante un material estable con gran porosidad, mediana capacidad de retención de agua y con microorganismos fijados, que puede ser utilizado para la degradación posterior de residuos orgánicos de fácil degradación.

\section{AGRADECIMIENTO}

Se agradece al Centro Modelo de Tratamiento de Residuos - CEMTRAR y al Centro de Investigación en Química, Toxicología y Biotecnología Ambiental - CIQTOBIA de la Universidad Nacional Agraria La Molina - UNALM, por el apoyo brindado.

\section{REFERENCIAS BIBLIOGRÁFICAS}

1. Wheeler E, Zajaczkowski JS. Horse Stable Manure Management.[Internet]. State college, Pennsylvania: Pennsylvania State University, College of Agricultural Sciences; 2009 [Citado agosto 2018]. Disponible en: http://panutrientmgmt.cas.psu.edu/pdf/G97. pdf.

2. Dagnino EP, Chamorro ER, Felissia FE, Area MC. Obtención de bietanol a partir de la celulosa presente en cascarilla de arroz y aserrín de algarrobo. Av Energ Renov Medio Ambient. 2014;18:06.01 - 06.07.

3. Piñeros-Castro Y, Velasco GA, Proaños J, Cortes W, Ballesteros I. Producción de azúcares fermentables por hidrólisis enzimática de cascarilla de arroz pretratada mediante explosión con vapor. Rev Ion. 2011;24(2):23-28.

4. Valverde GA, Sarria LB, Monteagudo YJ. Análisis comparativo de las características fisicoquímicas de la cascarilla de arroz. Sci Tech. 2007;(37):255-260.

5. Rynk R. On-Farm Composting Handbook. Printing Northeast Regional Agricultural Engineering Service. Itaca-NY: Natural Resource, Agriculture, and Engineering Service; 1992

6. Liebig J. Nuevas Cartas sobre la Química Considerada en sus aplicaciones a la Industria, a la fisiología y a la agricultura. Madrid: Imprenta de Don Agustin Espinosa y Compañía; 1853.

7. Huang GF, Wong JWC, Wu QT, Nagar BB. Effect of C/N on composting of pig manure with sawdust. Waste Manag. 2004;24(8):805-13.

8. Alexander M. Introduction to soil microbiology. Soil Sci. 1978;125(5):331.

9. Prochnow LI, Kiehl JC, Pismel FS, Corrente JE. Controlling ammonia losses during manure composting with the addition of phosphogypsum and simple superphosphate. Sci Agric. 1995;52(2):346-9.

10. Jiang J, Kang K, Chen D, Liu N. Impacts of delayed addition of N-rich and acidic substrates on nitrogen loss and compost quality during pig manure composting. Waste Manag. 2018;72:161-162 
11. Morisaki N, Chae Gun Phae, Nakasaki K, Shoda M, Kubota H. Nitrogen transformation during thermophilic composting. J Ferment Bioeng. 1989;67(1):57-61.

12. Bishop P, Godfrey C. Nitrogen transformations during sludge composting. Biocycle. 1983; 24: 34-39.

13. Jiang J, Liu X, Huang Y, Huang H. Inoculation with nitrogen turnover bacterial agent appropriately increasing nitrogen and promoting maturity in pig manure composting. Waste Manag. 2015;39:78-85.

14. El-Haddad ME, Zayed MS, El-Sayed GAM, Hassanein MK, Abd El-Satar AM. Evaluation of compost, vermicompost and their teas produced from rice straw as affected by addition of different supplements. Ann Agric Sci. 2014;59(2):243-251.

15. Van Ginkel. JT, Raats PAC, Van Haneghem IA Van. Bulk density and porosity distributions in a compost pile. Neth J. Agr Sci. 1999;47(2):105-121.

16. Lopez-Real J. Agro-industrial waste composting and its agricultural significance. Proceedings of the Fertilizer Society N²93. The Fertilizer Society, UK; 1990.p.3-26.

17. Kim E, Lee DH, Won S, Ahn H. Evaluation of optimum moisture content for composting of beef manure and bedding material mixtures using oxygen uptake measurement. AsianAustralasian J Anim Sci. 2016;29(5):753-758.

18. Kirchoff C, Malina J, Barrett M. Characteristics of Compost: moisture holding and water quality improvment. Center for Research in Water Resources. 2003;1-4.

19. Council U. Test methods for the examination of composting and compost. Rokonkoma, NY 2002.

20. Bernal MP, Alburquerque JA, Moral R. Composting of animal manures and chemical criteria for compost maturity assessment. A review. Bioresour Technol. 2009;100(22):5444-5453.

21. Binner E. $13^{\circ}$ Curso Internacional de Compostaje "Fundamentos, Operación y Diseño de Planta de Compostaje". Calidad y monitoreo del compost. Lima: Universidad Nacional Agraria La Molina; 2017.

22. Ö- NORM S 2023. pH Wert im Wasserextrakt BGBI,II. 2001;1778pp.

23. Bertoldi M, Vallini G, Pera A. The Biology of Composting: A Review. Waste Manag Res. 1983;1(1):157-176. 